Objectives We proposed to assess the incidence of varicocele in AS evaluated by physical examination and colour doppler ultrasonography (CDU). Sperm parameters of these patients were also investigated and compared with controls.

Methods Thirteen untreated male patients (ages 18-40) with AS and age matched healthy 12 male subjects (ages 18-39) included in the study. Testicular veins in both groups were studied with CDU and semen samples were analysed.

Results Eight of the patients evaluated with physical examination had varicoceles (all grade II, 5 left side, 3 bilateral). Only two subjects in control group had grade II varicocele on the left side and both were asymptomatic. Colour doppler evaluation of the testicular vessels in patients with AS revealed grade II varicocele (6 left side, 3 bilateral) in 9 of the patients $(69,2 \%)$ examined. Semen parameters were within normal limits in 7 patients, and there were altered sperm count and quality in 6 patients.

Conclusion The high incidence of varicocele in patients with AS may be explained by chronic inflammatory low-back pain that brings about reduced extensor muscle strength that causes compensatory increase in intra-abdominal pressure. This may cause a mechanical overload to the testicular vessels. Secondly, vasodilator effect of nitric oxide that was activated by inflammatory process.

\section{SAT0022 SUCCESSFUL TREATMENT OF PATIENTS WITH SEVERE UNDIFFERENTIATED SPONDYLOARTHROPATHY WITH THE ANTI-TNFA ANTIBODY INFLIXIMAB}

1J Brandt, ${ }^{2} \mathrm{H}$ Haibel, ${ }^{3} \mathrm{~J}$ Sieper, ${ }^{4} \mathrm{~J}$ Braun. ${ }^{1}$ Nephrology; ${ }^{2}$ Gastroenterology/Rheumatology; ${ }^{3}$ Gastroenterology/Rheumatology, German Rheumatology Research Center, Benjamin Franklin Hospital, Free University, Berlin; ${ }^{4}$ Rheumatology, Rheumazentrum Ruhrgebiet, Herne, Germany

\subsection{6/annrheumdis-2001.374}

Background Anti-TNFa therapy has been successfully used in patients with active ankylosing spondylitis (AS;A\&R June 2000). Whether this treatment is also efficacious in patients with undifferentiated spondyloarthropathies (uSpA), the second most frequent $\mathrm{SpA}$ subset, has not been assessed to date.

Objectives To test the efficacy of the monoclonal anti-TNFa antibody infliximab in patients with severe uSpA.

Methods Six uSpA patients (4 male, 2 female) with a median age of 36 years (range 19-49) were diagnosed according to the 1991 ESSG criteria. The median disease duration was 6.2 years (1.235 years). They had been refractory to other therapies including NSAIDs, steroids and sulfasalazine and were treated with infliximab in an open observational way at week 0,2 and 6 and followed up for 12 weeks. Three patients received infliximab in a dosage of $3 \mathrm{mg} / \mathrm{kg}$ and the other three received $5 \mathrm{mg} / \mathrm{kg}$. The leading clinical symptoms were peripheral arthritis $(n=6)$, inflammatory back pain $(\mathrm{n}=6)$ and enthesitis of the heels $(\mathrm{n}=$ 4). HLA B27 was positive in $5 / 6$ patients. No patient had sacroiliitis $>$ grade II unilateral. The Bath AS disease activity index (BASDAI), the functional index (BASFI), pain on a visual analogue scale (VAS), and the Bath AS Metrology Index (BASMI) were assessed before, during and after therapy. Quality of life was measured using the Short Form (SF) 36 instrument.

Results All patients improved after 1-3 days. There was a more substantial response in the group treated with $5 \mathrm{mg} / \mathrm{kg}$ infliximab then in the $3 \mathrm{mg} / \mathrm{kg}$ group. The range of the BASDAI in the 5 $\mathrm{mg} / \mathrm{kg}$ group was $7.0-7.6$ (3 $\mathrm{mg} / \mathrm{kg}$-group: $7.7-8.6)$ before and 6 weeks after the third infusion $0.7-2.7$ (3 mg/kg-group: $1.7-$ 7.8). The functional index also improved in all but one patient: the BASFI values had decreased from $7.0-7.6$ in the $5 \mathrm{mg} / \mathrm{kg}$ group (3 mg/kg-group: $6.7-8.5$ ) to $0.8-2.7$ (3 mg/kg-group: $0.8-$ 8.4). Pain on a VAS improved in the $5 \mathrm{mg} / \mathrm{kg}$ group from $7.1-$ 7.7 (3 mg/kg-group: $5.1-9.0$ ) to $0.8-2.6$ (3 mg/kg-group: $1.2-$ 8.1). Joints, entheses and spinal symptoms improved equally. CRP levels dropped from $<6.0-120 \mathrm{mg} / \mathrm{l}$ before therapy to $<6.0-15.6 \mathrm{mg} / \mathrm{l}$ at week 12 . No side effects occurred. There was improvement in all 8 SF-36 concepts assessed at baseline and at week 12; the improvement was significant for the concepts bodily pain and vitality.

Conclusion These data suggest that anti-TNFa therapy has significant short-term efficacy in severe uSpA. Infusions with $5 \mathrm{mg} / \mathrm{kg}$ infliximab might be more effective than $3 \mathrm{mg} / \mathrm{kg}$ in $S p A$ patients. Since it is known that $30-50 \%$ of uSpA patients develop AS over time it seems worthwhile to study whether this can be prevented by anti-TNFa therapy.

\section{SAT0023 HLA GENE POLYMORPHISM IN PSORIATIC ARTHRITIS (PSA)}

${ }^{1} \mathrm{~S}$ Perrot-Andre, ${ }^{2} \mathrm{~A}$ Ramouneau-Pigot, ${ }^{2} \mathrm{Y}$ Fontana, ${ }^{1} \mathrm{~J}$ Sany, ${ }^{2} \mathrm{JF}$ Eliaou, ${ }^{1} \mathrm{~B}$ Combe. ${ }^{1}$ Rhumatologie; ${ }^{2}$ Immunologie, Lapeyronie, Montpellier, France

10.1136/annrheumdis-2001.375

Background Psoriasis is known to be associated with HLA component (HLA-B*13, B*17, Cw*06 and DRB1*07). However few data exist on HLA association with PsA especially using highly sensitive HLA-Cw, DR and DQ typing methods.

Objectives The aim of this work was to study the distribution of HLA alleles in PsA patients in comparison with unrelated controls from the same geographic area.

Methods 69 patients (35 males, 34 females) were included according to modified Avila criteria. A first control group was composed of 328 healthy individuals (random controls: RC). Because alleles at the $\mathrm{B}$ and $\mathrm{Cw}$ loci are in strong linkage disequilibrium, 60 control individuals matched with patients for HLA-B alleles were selected (matched controls: MC). All the individuals were typed for HLA-A and B by microlymphocytoxicity and for HLA-Cw, DRB1 and DQB1 by PCR-SSP after geno-mic DNA extraction of peripheral blood nucleated cells. The significance of differences in phenotype frequencies was determined by Fischer's exact test.

Results No significant difference in the HLA-B allele distribution exists between patients and MC. Disease expression of the PsA patients was as follows: skin psoriasis in 100\%, axial PsA in $37.7 \%$, asymmetric oligoarthritis in $15.9 \%$, symmetric polyarthritis in $34.8 \%$ and asymmetric polyarthritis in $46.4 \%$ of the patients. HLA-A*01 ( $\mathrm{p}=0.0011), \mathrm{B} * 27(\mathrm{p}=0.03), \mathrm{B} * 38(\mathrm{p}=$ $0.003)$ and $B * 57(p=0.03)$ were significantly increased, whereas HLA-A*11 $(\mathrm{p}=0.017)$ and $\mathrm{B} * 18(\mathrm{p}=0.0019)$ were significantly decreased in PsA patients compared to RC. $34.4 \%$ of the PsA patients carried $\mathrm{Cw}^{*} 0602$ compared to $11 \%$ in RC $(p=0.003)$. No difference of DRB1 and DQB1 allele frequencies could be evidenced. When PsA patients and MC were compared no difference of HLA class I and class II allele frequencies was shown except for a significant increase of $\mathrm{Cw}^{*} 0102$ in patients ( $p=0.0025$ ). Early onset of PsA (as well as psoriasis) was significantly associated with $\mathrm{Cw}^{*} 0602(\mathrm{p}=0.01)$ and DRB1 07 ( $p=0.03$ ). No association between spondilytis (axial PsA) with and B*27 was observed. Finally, although the sample size is small (10 patients), severe forms of disease were significantly associated with HLA-B*38 ( $\mathrm{p}=0.0035)$. 